\title{
Response to comment on "Solid Recovered Fuel: Materials Flow Analysis and Fuel Property Development during the Mechanical Processing of Biodried Waste"
}

\author{
Costas A. Velis ${ }^{1,2}$, Stuart Wagland ${ }^{1}$, Phil Longhurst ${ }^{1}$, Bryce Robson ${ }^{3}$, Keith Sinfield ${ }^{3}$, \\ Stephen Wise $e^{3}$ and Simon Pollard ${ }^{1 *}$ \\ ${ }^{1}$ Cranfield University, Centre for Energy and Resource Technology, Department of \\ Environmental Science and Technology, School of Applied Sciences, Cranfield, Bedfordshire \\ MK43 0AL, UK; \\ ${ }^{2}$ University of Leeds, School of Civil Engineering, Leeds, LS2 9JT, UK; \\ ${ }^{3}$ Shanks Waste Management Ltd, Dunedin House, Mount Farm, Milton Keynes, \\ Buckinghamshire, MK1 1BU, UK \\ *Corresponding author, phone: +44 1234 754101, fax: +44 1234 754036, email: \\ s.pollard@cranfield.ac.uk
}

Laner and Cencic ${ }^{1}$ comment on Velis et al. $(2013)^{2}$ clarifying certain points on the use of the material flow analysis (MFA) software $\mathrm{STAN}^{3}$. We welcome the correspondence and the opportunity this exchange provides to discuss optimal approaches to using STAN. In keeping with Velis et al. ${ }^{2}$ these physically impossible, and otherwise insignificant, negative flows have enabled improvements to STAN. Here, we elaborate on the practicalities of using STAN in our research and on the correctness and validation of our results, notwithstanding the inclusion of negative flows. We explain the contribution of our approach to solid waste management and resource recovery.

To our knowledge, discussion about physically impossible flows resulting from reconciliation in STAN was for the first time raised here ${ }^{2}$. Laner and Cencic" state that "occurrence of negative flows [while applying STAN] indicates problems with input data, as contradictions in the mass balance equations lead to unrealistic results after data reconciliation." They also refer to "different approaches [...] to handle uncertainties in MFA, differing in [...] sophistication, mathematical rigor, and applicability". We agree that detailed information and analysis on such approaches is missing and could enable the improved application of MFA software. We also contend that data quality in Velis et al. ${ }^{2}$ employs best laboratory practice, as identified in the methodology sections ${ }^{2,4}$, accounting for the nature of sampling MBT plants that treat municipal solid waste ${ }^{5}$ (highly heterogeneous, dynamically evolving flows sampled with time differentials from hours to days). High between- 
sample variability and conflicting data between sampling points is a practical reality for waste systems, which is the core challenge addressed ${ }^{2}$ that renders the application of data reconciliation software necessary. We purposefully selected STAN, built with such applications in mind": "But due to measurement uncertainties contradictions in input data are inevitable. Often it is not clear how to handle these inconsistent data. STAN offers the possibility to reconcile these values in order to find the best fitting ones without guessing".

Because of the negative flows, Laner and Cencic ${ }^{1}$ are incidentally concerned about the validity of our results and suggest corrections, i.e. setting to zero the uncertainties for the problematic flows. Elsewhere, they speculate the problem stems not from the large uncertainties, but from "model inconsistencies" (not further specified). Their run of the model is not sufficiently explained, but possibly re-allocates some/all of the negative flows as positive flows to the solid recovered fuel (SRF) production stream. Notably, this suggestion is mathematical and not necessarily founded on a process understanding of the modelled system, and may not be the optimal way to reconcile the system if the small negative flows were to be eliminated. In any case, they recognise their approach would result only in minimal changes. Their 'corrected' values for transfer coefficients (TC) to the SRF output differ insignificantly from those reported ${ }^{2}$ (from $0.2 \%$ to $3.4 \%$ ) and do not affect the representativeness of the results and their engineering significance. In all cases, the correction in the arithmetic mean is within the limits of the reported uncertainty $\left(\mathrm{U}_{95}, 95 \%\right.$ confidence interval) (Table $1)^{2}$ and typically a fraction of those: (TC total chlorine: $80.0 \pm 26.2$, instead of $78.9 \pm 26.2$; TC net calorific value: $74.3 \pm 8.6$ instead of $73.2 \pm 8.6$; $\mathrm{TC}$ ash content $39.1 \pm 3.1$, instead of $37.8 \pm 3.1$ ). To illustrate, the amount in Velis et al. ${ }^{2}$ deducted from the flow contributing to the SRF output, due to the negative flow, is comparatively zero from an engineering point of view: $0.4 \%$ of the SRF $\mathrm{Cl}$ output ( 0.34 units not counted for an output of $78.3 \pm 6.0$ ), especially considering the uncertainty $\left(\mathrm{U}_{95}\right)$ on the arithmetic mean (amount not counted is $2.8 \%(=0.34 /(2 * 6.0) * 100)$ of the $\left.\mathrm{U}_{95}\right)$. The same applies to the other properties discussed ${ }^{1}$ and other properties reported ${ }^{2}$. To put these in context, the suggested ${ }^{1}$ corrections are infinitesimal compared to the data input inconsistencies inherent to waste MFA": "it is very rare that a balance between inputs and outputs of a measured system yields an error less than $10 \%$ of the total flow" and "Balance differences between input and output of $10 \%$ are common and are usually not significant for the conclusions." Hence, we contend they cannot form the basis for criticism.

Laner and Cencic ${ }^{1}$ note that "Data refinement and analysis is naturally an iterative procedure and a crucial step in mass flow analysis [MFA]". They advise that modellers should check the plausibility of STAN outcomes and manually correct. Our iterative methodology applies such good practice and reality checks on STAN outputs. The plausibility of outcomes was assessed and, where the suggested reconciled flows were not feasible, corrected. Velis et al. ${ }^{2}$ and the related Supporting Information (SI) provided detail on the MFA procedures and data handling. Results were validated to the stated degree of uncertainty by a series of cross-checking. First, data reconciliation and calculation of TCs using STAN was attempted only after confirming the closing of overall input vs. the sum of output balances, and detailed sorting analysis of input/ outputs $\left(25\right.$ items - Table $\left.S 1^{4}\right)$. Second, the negative flows appearing around the Fe buffering point are of minor concern, because the main flow of relevance is the ferrous materials, for which a fully balanced MFA was achieved (Figure 1). Third, flow component (waste) chemical properties were used to successfully model SRF chemical properties ${ }^{4}$. Fourth, successful balancing of the combustible dry-ash free matter suggests the mutual compatibility of three independently-obtained data sets (total as received mass, moisture content per component, ash content per component) ${ }^{2}$. 


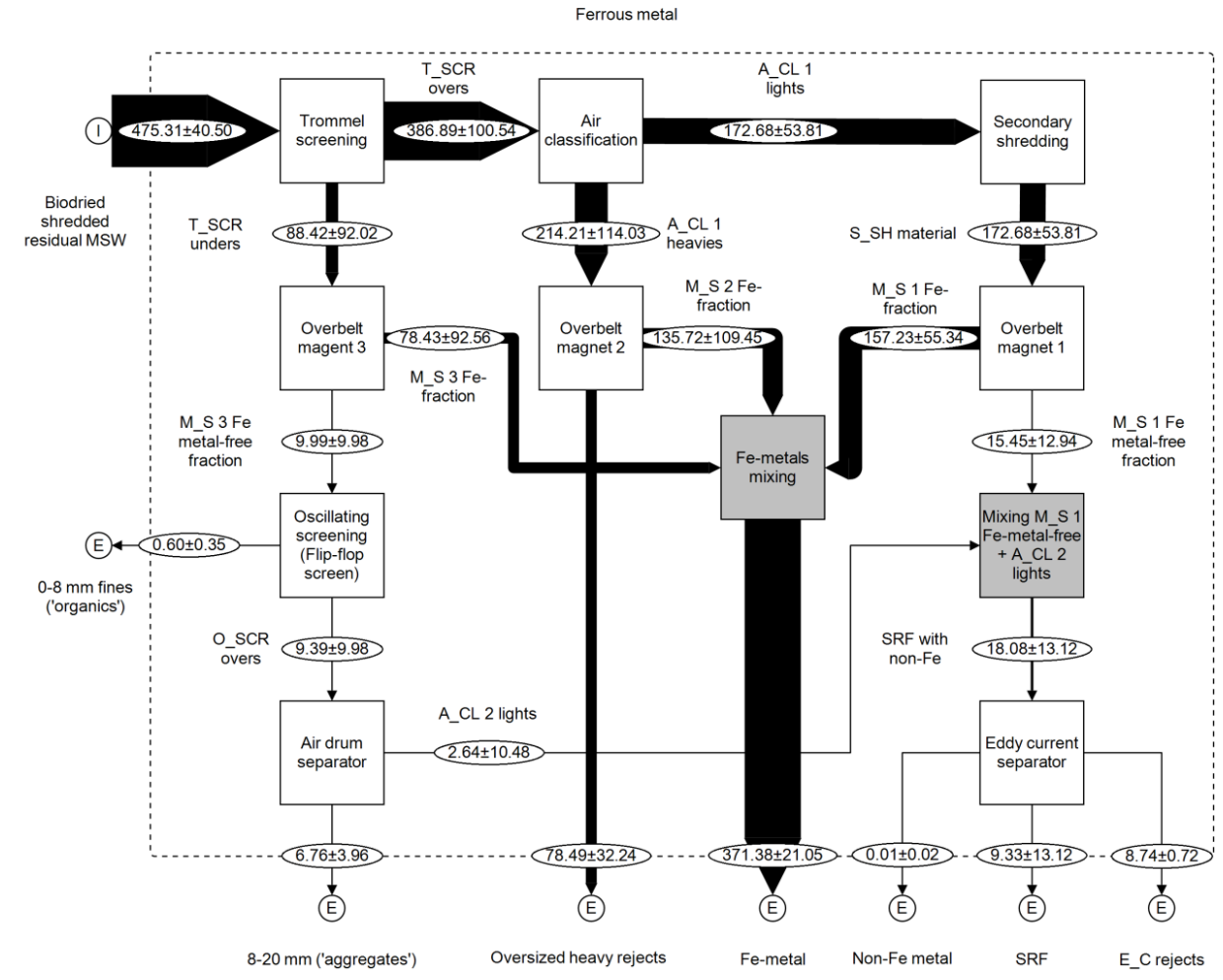

Figure 1. Sankey diagram of the processing section of the UK biodrying MBT plant modelled in Velis et al. ${ }^{2}$ : ferrous metal waste component model of flows. Values are average specific (per component) mass load $\left({ }_{\mathrm{ar}}\right)$, out of ca 10,000 overall components mass input, plus/minus the total extended uncertainty $\left(\mathrm{U}_{95}\right)$ (confidence intervals around the average at $95 \%$ confidence).

Our view is that having mathematically zeroed these negative flows, or the uncertainties around them, would have concealed or mislead $E S \& T$ readers about the precision of flows. The benefits of maintaining this information is that it raises awareness, opens this debate and faithfully informs readers about the uncertainty around these highly heterogeneous flows. It may be useful for future editions of STAN to provisionally prevent physically impossible negative flows, if compatible with the data reconciliation algorithms, and resolve such cases with automation rather than by manual correction. In concluding, Velis et al. ${ }^{2}$ describes the most detailed application of STAN to date for a challenging case of gross sample heterogeneity among waste inputs, providing novel insights on the performance of SRF-producing MBT plants and iterative MFA solutions for all solid waste processing plants. This can only accelerate the application of manufacturing know-how to waste processing ${ }^{8}$.

(1) Laner, D.; Cencic, O., Comment on "Solid recovered fuel: materials flow analysis and fuel property development during the mechnical processing of biodryied waste". Environ. Sci. Technol. 2013.

(2) Velis, C. A.; Wagland, S.; Longhurst, P.; Robson, B.; Sinfield, K.; Wise, S.; Pollard, S., Solid recovered fuel: Materials flow analysis and fuel property development during the mechanical processing of biodried waste. Environ. Sci. Technol. 2013, 47, (6), 2957-2965.

(3) Institute for Water Quality Resources and Waste Management TU-Wien STAN2.0, Software for substance flow analysis; Institute for Water Quality, Resources and Waste Management, Vienna University of Technology 2009. 
(4) Velis, C. A.; Wagland, S.; Longhurst, P. J.; Robson, B.; Sinfield, K.; Wise, S.; Pollard, S. J. T., Solid recovered fuel: the influence of waste stream composition and processing on chlorine content and fuel quality Environ. Sci. Technol. 2012, 46, (3), 1923-1931.

(5) Skutan, S.; Brunner, P. H., Metals in RDF and other high calorific value fractions from mechanical treatment of MSW: analysis and sampling errors. Waste Manage. Res. 2012, 30, (7), 645655 .

(6) Cencic, O.; Rechberger, H., Material flow analysis with software STAN. Journal of Environmental Engineering and Management 2008, 18, (1), 3-7.

(7) Brunner, P. H.; Rechberger, H., Practical handbook of material flow analysis. Lewis Publishers: Washington, D.C., US, 2004.

(8) Velis, C. A.; Cooper, J., Are solid recovered fuels resource-efficient? Waste Manage. Res. 2013, 31, (2), 113-114. 